\title{
Centro Cultural de Saramenha: integrando a universidade à comunidade de Ouro Preto
}

DOI: 10.37702/2175-957X.COBENGE.2021.3562

José Matheus Vieira Matos - matheus.vieira225@gmail.com

Universidade Federal de Ouro Preto

Rua João Fernandes Vieira 256

35400-000 - Ouro Preto - MG

Anays Cristina Guimarães Dias - anayscristina@yahoo.com.br

Universidade Federal de Ouro Preto

Rua: José Mendes 235

35400-000 - OURO PRETO - MG

Carlos Alberto Pereira - pereiraufop@gmail.com

Universidade Federal de Ouro Preto

Rua João XXIII 240

35400-000 - Ouro Preto - MG

Resumo: O Centro Cultura de Saramenha é fruto de uma ação conjunta entre a prefeitura municipal de Ouro Preto, a UFOP - Departamento de Engenharia de Minas e os moradores do bairro Saramenha. O Centro conta com uma biblioteca comunitária, cadastrada na Fundação Biblioteca Nacional e possui um acervo de aproxima-damente 4000 livros, atualizados à disposição da comunidade, um salão de festas, uma cozinha e uma quadra de grama sintética. $O$ espaço permite a participação da comunidade em diversas atividades culturais, desenvolvidas por voluntários e graduandos da UFOP, que visam combater a exclusão informacional e proporciona um local de convivência, ação popular, produção e disseminação de cultura e conhecimento para os moradores do bairro e de seu entorno. Tais atividades, como o estímulo à leitura, auxílio no dever de casa e pesquisa, oficinas diversas, aulas de dança, brincadeiras lúdicas, rodas de conversa, empréstimos de livros, jogos, reforço escolar e aulas de idiomas atraem a atenção da comunidade e transformam o Centro em uma ferramenta de apoio didático pedagógico e de difusão cultural para as crianças, jovens e adultos. Desta forma o Centro Cultural cumpre o seu papel como espaço de convívio social e amplifica as margens de aprendizado de todos os envolvidos. Aos moradores, dando-lhes oportunidades antes não oferecidas e aos graduandos uma rica oportunidade de crescimento 


\section{COBENGE DEvento online

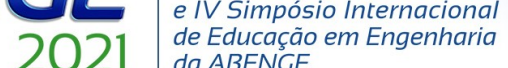 \\ 28 a 30 de SETEMBRO \\ "Formaçāo em Engenharia: Tecnologia, Inovaçâo e Sustentabilidade"}

humano e profissional.

Palavras-chave: Extensão. Engenhosidade. Cultura. Comunidade. 


\section{CENTRO CULTURAL DE SARAMENHA: INTEGRANDO A UNIVERSIDADE À COMUNIDADE DE OURO PRETO}

\section{INTRODUÇÃO}

Segundo Ramos (2008) a ideia dos centros culturais surgiu na segunda metade do século XX. Países como Inglaterra e França passaram a criar e incentivar a implantação de espaços culturais na tentativa de democratizar a cultura para além das tendências da cultura de massa, não demorando muito para que diversos países copiassem a iniciativa pioneira. No Brasil não foi diferente, entretanto a história de Centros Culturais brasileiros é recente, embora já houvesse interesse em sua criação desde a década de 1960, sendo que os primeiros surgiram na década de 1980 com os nomes de Centro Cultural do Jabaquara e Centro Cultural de São Paulo, ambos localizados no estado de São Paulo.

Segundo Cardoso e Nogueira (1994), o impulso à criação dos espaços culturais se deu a medida do entendimento de que a cultura é feita no cotidiano da existência dos homens e não somente pela explosão informacional da contemporaneidade.

O Centro Cultural de Saramenha não foi criado inicialmente como Centro Cultural, mas sim como biblioteca comunitária. A implantação se iniciou no ano 2005, com o projeto de extensão Oficina de Ciência e Cidadania, onde fundou a biblioteca comunitária no Morro São Sebastião no ano de 2002. Com o sucesso dessa biblioteca o Departamento de Engenharia de Minas da Universidade Federal de Ouro Preto - UFOP, juntamente com o Instituto Federal de Minas Gerais - IFMG, a Prefeitura Municipal de Ouro Preto, a Associação de Moradores do bairro Saramenha e representantes da Escola Municipal Renê Giannetti decidiram expandir o projeto, criando uma biblioteca comunitária no bairro Saramenha.

As bibliotecas comunitárias são ambientes físicos que possuem um acervo bibliográfico multidisciplinar, normalmente não possuem intervenção do poder público, são mantidas pela comunidade civil e em alguns casos são mantidas por entidades externas. Segundo Machado e Vergueiro (2010) elas funcionam como ponto irradiador de cultura, e geralmente são criadas por iniciativa individual ou coletiva em regiões periféricas e trazem um lado humano forte, pois são espaços livres onde além do empréstimo de livros e espaço para leitura acontecem reforço escolar, oficinas e atividades recreativas.

Segundo Cavalcante (2011), possuem a finalidade de ajudar a comunidade a enfrentar as dificuldades que surgem no cotidiano, pela falta de acesso à informação e à leitura. A biblioteca comunitária de Saramenha funcionou até o ano de 2016. A partir do ano de 2017 passou a ser considerada Centro Cultural de Saramenha, uma vez que a biblioteca foi repensada e expandida.

Pertencentes à esfera municipal, a maioria das bibliotecas tem ação que ultrapassam suas obrigações tradicionais, mantendo projetos para públicos específicos, tais como os de estímulo à leitura, voltado para crianças, assim como projetos para terceira idade. Desenvolvem, ao mesmo tempo, uma gama de atividades ligadas às artes (dança, música, teatro, por exemplo). Algumas têm um núcleo Braille, outras mantém sessões semanais de cinema, numa região em que não há nenhuma sala cinematográfica comercial. Ou seja, percebe-se um esforço de se responder a demandas mais amplas do que simplesmente colocar livros à disposição de consulentes, funcionando, em alguns casos, como pequenos centros 
culturais. (Botelho, 2003).

\section{OBJETIVO GERAL}

O projeto realizado no Centro Cultural de Saramenha tem como principal objetivo suprir a necessidade de informação, cultura e lazer das crianças, adolescentes e adultos. Esse ambiente de compartilhamento, troca e fluxos de informações funciona como um instrumento de inclusão informacional dentro da comunidade e proporciona o crescimento pessoal dos cidadãos através de práticas como as atividades de leitura.

\subsection{Objetivos Específicos}

Desta forma, o centro cultural tem como objetivos principais:

- valorizar a comunidade do bairro;

- integralizar os cidadãos na sociedade da informação;

- combater a exclusão informacional e proporcionar um local de convivência, ação popular, produção e disseminação de cultura e conhecimento;

- estimular o interesse da comunidade na leitura, aprendizado e exercício da cidadania;

- melhorar o desempenho escolar das crianças, jovens e adultos;

- ampliar os conhecimentos acerca dos cursos de engenharia oferecidos na universidade;

- assegurar o acesso fácil e gratuito a livros multidisciplinares, cursos e oficinas em um local seguro de interação e lazer, além de integrar os alunos da universidade e comunidade no intuito de formar leitores e cidadãos bem informados.

\subsection{Justificativa}

O bairro Saramenha de Cima, como outros próximos, é desprovido de mecanismos relacionados à disseminação da informação, do acesso à leitura e ao livro. Nesse contexto, fica evidente a necessidade de criação de políticas sociais que atuem diretamente na minimização das lacunas da desigualdade social, informacional e cultural dentro da comunidade.

Deste modo, a implantação de uma biblioteca comunitária local, atua como facilitador social e de apoio e promoção às atividades de caráter didático-pedagógico, intelectual e social. Além disso, o projeto atende a outros membros da comunidade residentes em bairros localizados próximos do Saramenha de Cima, ampliando os limites alcançados pelas atividades desenvolvidas na biblioteca. Na "Figura 1", tem-se o primeiro grupo atendido pelo projeto.

Figura 1 - Equipe de voluntários e bolsistas e parte das crianças atendidas do bairro Saramenha. 


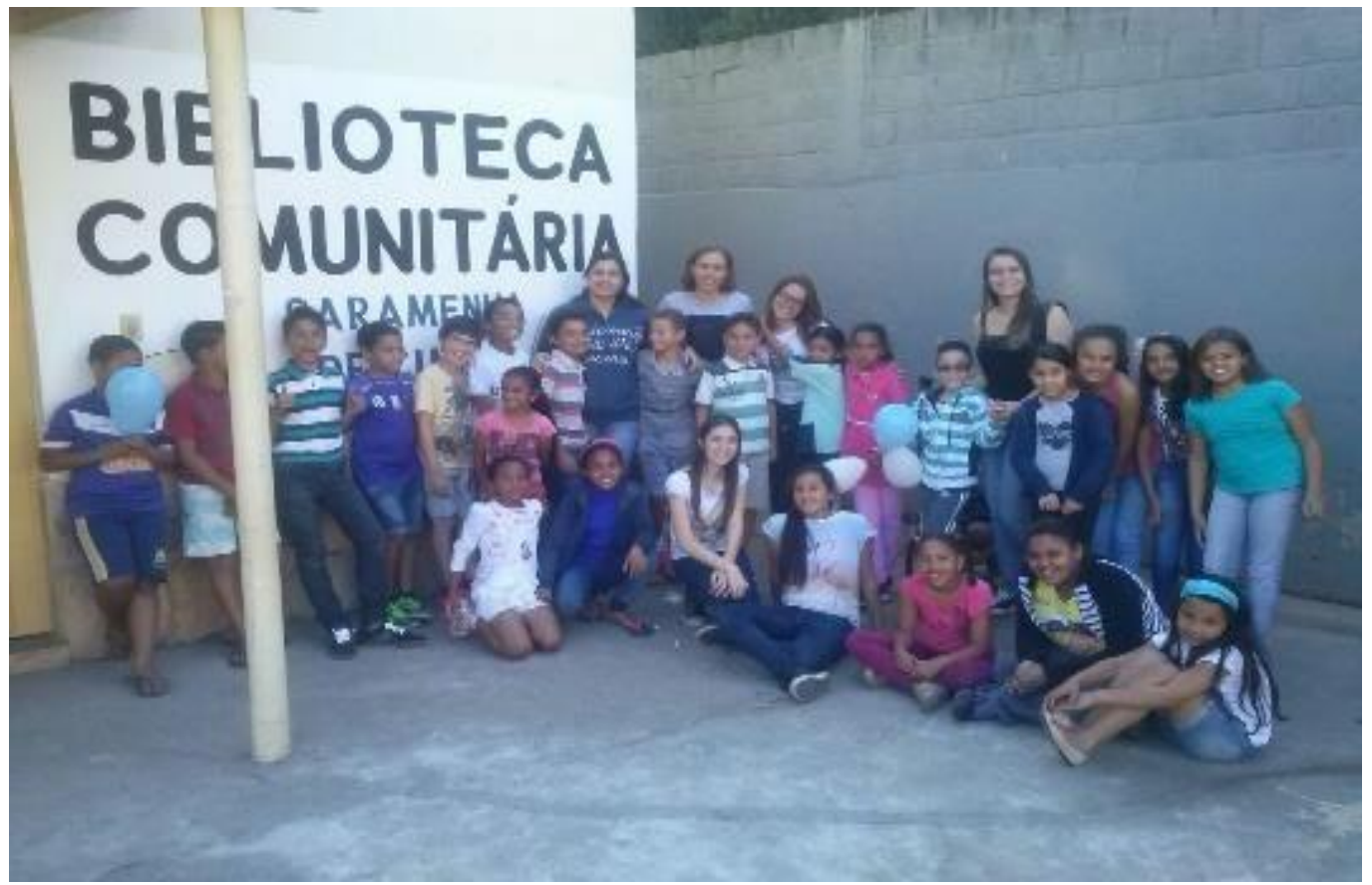

Fonte: elaborada pelo autor.

\subsection{Atividades Desenvolvidas}

É oferecida uma grande variedade de livros para pesquisa e empréstimos, atividades relacionadas à leitura, reforço escolar e aulas da disciplina de inglês, disponibilizadas para qualquer faixa etária. Iniciou-se um curso piloto de francês, objetivando a introdução do conhecimento sobre a cultura de países francófonos, focando no desenvolvimento da conversação, escrita e leitura no idioma, cujo formato será utilizado também em um curso de Inglês com a mesma finalidade.

São oferecidas também a oficina de jogos e brincadeiras e a de dança, apresentadas a todos da comunidade, visando promover práticas culturais, contribuir na construção da personalidade e desenvolver a noção de equilíbrio e espaço, contribuindo inclusive no desenvolvimento das atividades motoras e da expressão corporal.

Além disso, realizou-se semanalmente a reunião da equipe para planejamento das ações, avaliação das atividades e atualização, definição do material necessário para as atividades, revisão bibliográfica e mensalmente a reunião com o coordenador, orientadores, bolsistas e voluntários.

\section{METODOLOGIA}

A gestão do espaço e planejamento das ações é de responsabilidade do coordenador do projeto de extensão da UFOP, da presidência da Associação de Moradores, juntamente com um representante da Escola Municipal Renê Giannetti. O centro também conta com o trabalho de discentes voluntários/bolsistas e um grupo formado por professores e técnicos administrativos da UFOP e do IFMG, bem como mestrandos do Programa de Pós-Graduação em Engenharia Mineral - PPGEM/DEMIN.

O ambiente funciona de segunda a sexta-feira, das $13 \mathrm{~h}$ às $17 \mathrm{~h}$, horário de maior circulação de crianças e jovens no local, possuindo uma programação variada e criativa 
que instiga a curiosidade da comunidade presente. O público que frequenta o projeto incluí desde crianças que buscam ajuda na lição de casa, aulas de idiomas e/ou momentos de lazer e recreação, até adultos e idosos que buscam empréstimos de livros, participação nas oficinas e rodas de discussões.

O acervo de livros da biblioteca do centro é, em sua maioria, proveniente de recursos vindos dos editais da Novelis, Ministério da Educação, doações feitas pela própria comunidade e alguns livros, geralmente best-sellers ou lançamentos, comprados com verba de doações. Para a organização do acervo, utilizou-se um software gratuito chamado BookDB2, que possibilitou a catalogação, gerenciamento e cadastro de usuários, controlando a entrada e saída dos livros. Para manter a organização e conservação dos mesmos, os bolsistas fazem uma seleção a cada 6 meses dos que se encontram em pior estado para reformas (encadernação e limpeza).

No calendário de 2020/21 a biblioteca comunitária contou com uma programação variada, planejada e adaptada visando atender o maior público possível. As atividades realizadas com mais ênfase durante esse período foram:

- Empréstimo de livros: a biblioteca do centro cultural possui um grande acervo, totalizando 4.000 livros, que foram disponibilizados aos usuários para consultas no local ou para empréstimo com o prazo de uma semana para devolução;

- Auxílio no dever de casa e na pesquisa escolar: os bolsistas da biblioteca disponibilizaram um horário de segunda a quinta das $13 \mathrm{~h}$ às $15 \mathrm{~h} 30 \mathrm{~min}$ para auxílio aos usuários em suas tarefas escolares;

- Reforço escolar: no auxílio do dever de casa foi possível notar a dificuldade de cada criança. Algumas dificuldades puderam ser esclarecidas durante o auxílio do dever de casa, mas quando necessário a criança foi encaminhada ao reforço escolar, que aconteceu durante as terças e quintas-feiras das $15 \mathrm{~h}$ às $17 \mathrm{~h}$;

- Exercícios de aprendizagem e memorização: foram disponibilizados jogos de tabuleiros, quebra-cabeças, jogo da memória, jogo dos sete erros, e outros jogos de mesa, oferecendo lazer, interação social e ao mesmo tempo maior aprendizagem e desenvolvimento das habilidades cognitivas dos usuários;

- Inclusão no mundo digital: houve disponibilidade de um computador para os usuários proporcionando maior contato tecnológico, e familiarização com ferramentas computacionais. Mesmo sem acesso à internet, os computadores foram utilizados para digitalização de pesquisas escolares, jogos didáticos e gerenciamento do acervo literário.

- Oficinas: o centro elaborou um cronograma de oficinas que seriam realizadas durante o ano de 2020, seguindo o calendário escolar dos alunos, e de acordo com cada data comemorativa. Devido à pandemia da Covid-19 as atividades foram suspensas e não foi possível a realização do cronograma planejado para o período.

- Oficina de leitura: foi realizada todas as quartas-feiras, de forma remota, e teve o objetivo de desenvolver a habilidade de leitura e interpretação de texto dos participantes. Eram sorteados títulos literários de acordo com a faixa etária e o gosto das crianças presentes, em seguida uma criança se disponibilizava a ler enquanto as demais prestavam atenção. Após a leitura, uma roda de discussão era aberta onde os participantes tinham a oportunidade de expor seu ponto de vista sobre a leitura, em seguida fazia-se uma atividade de perguntas e respostas sobre o texto, permitindo a avaliação do nível de interpretação de cada leitor. Ao fim das atividades os participantes recebiam uma pontuação e um prêmio pelo seu desempenho;

Devido à pandemia da Covid-19 as atividades do centro cultural foram paralisadas nos meses de abril a novembro de 2020. Durante esse período o prédio onde está localizado o centro comunitário também entrou em reforma por causa de 
um vazamento no pavimento superior ao espaço onde está sediada a biblioteca.

Desta forma os discentes trabalharam de maneira remota durante este período, planejando as oficinas de dança, artesanato e desenho, criando material de estudos para as aulas de idiomas e elaborando uma conta na rede social Instragram para interagir com a comunidade, publicar novidades com relação às atividades oferecidas e vídeos informativos.

Além disso eles atenderam a comunidade pelo aplicativo WhatsApp. Assim, conseguiram realizar alguns empréstimos de livros e auxiliaram de maneira remota alguns alunos da Escola Municipal Tomas Antônio Gonzaga, quando eram solicitados pelo aplicativo. Em novembro o acervo da biblioteca foi catalogado e os livros organizados. O espaço recebeu algumas pinturas e decorações para reabertura em dezembro. Na "Figura 2" pode-se observar como estava o espaço antes da reforma, e na "Figura 3" o mesmo após a conclusão dos preparativos para reabertura.

Figura 2 - Espaço onde se realizava o projeto antes da reforma.

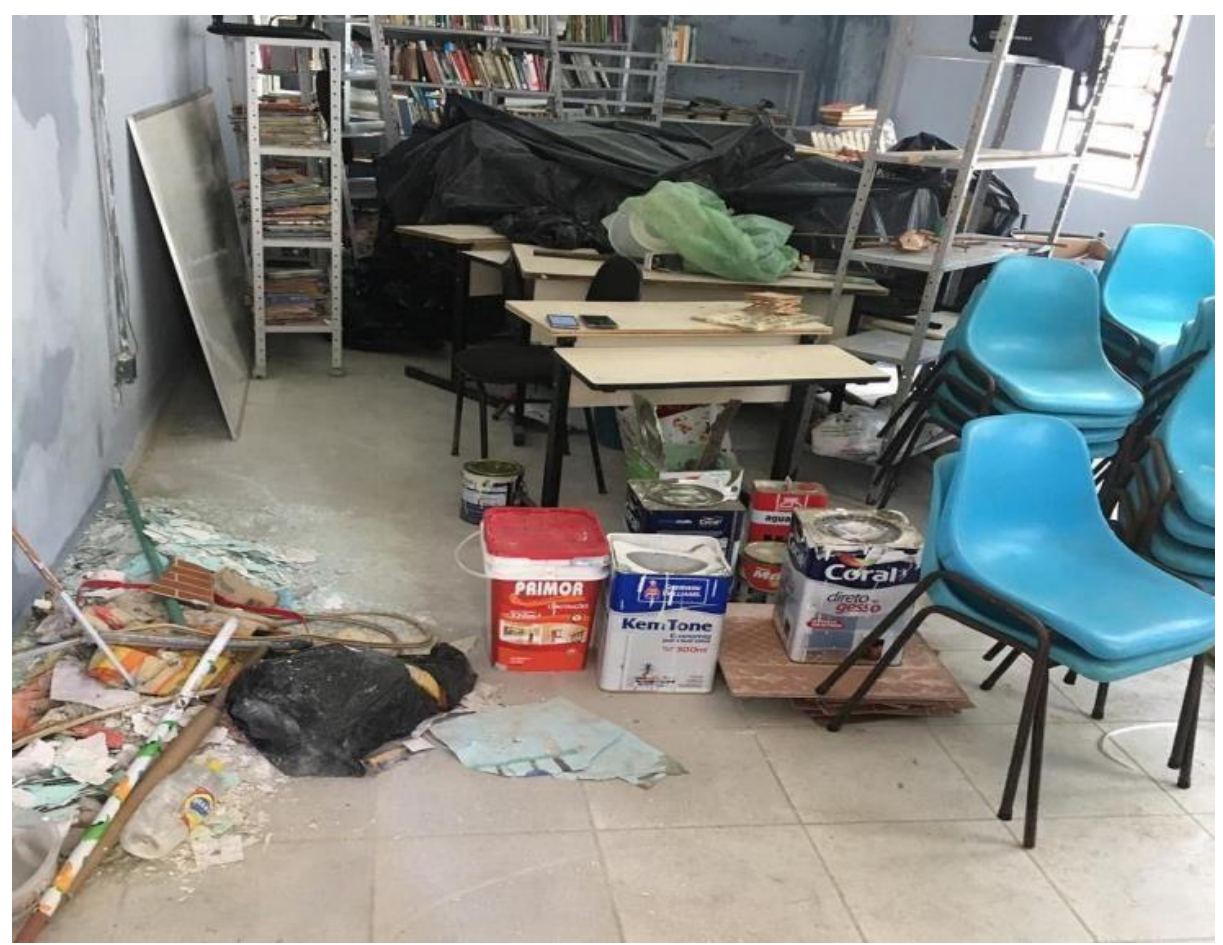

Fonte: elaborada pelo autor. 
Figura 3 - Espaço onde se realiza o projeto após a reforma.

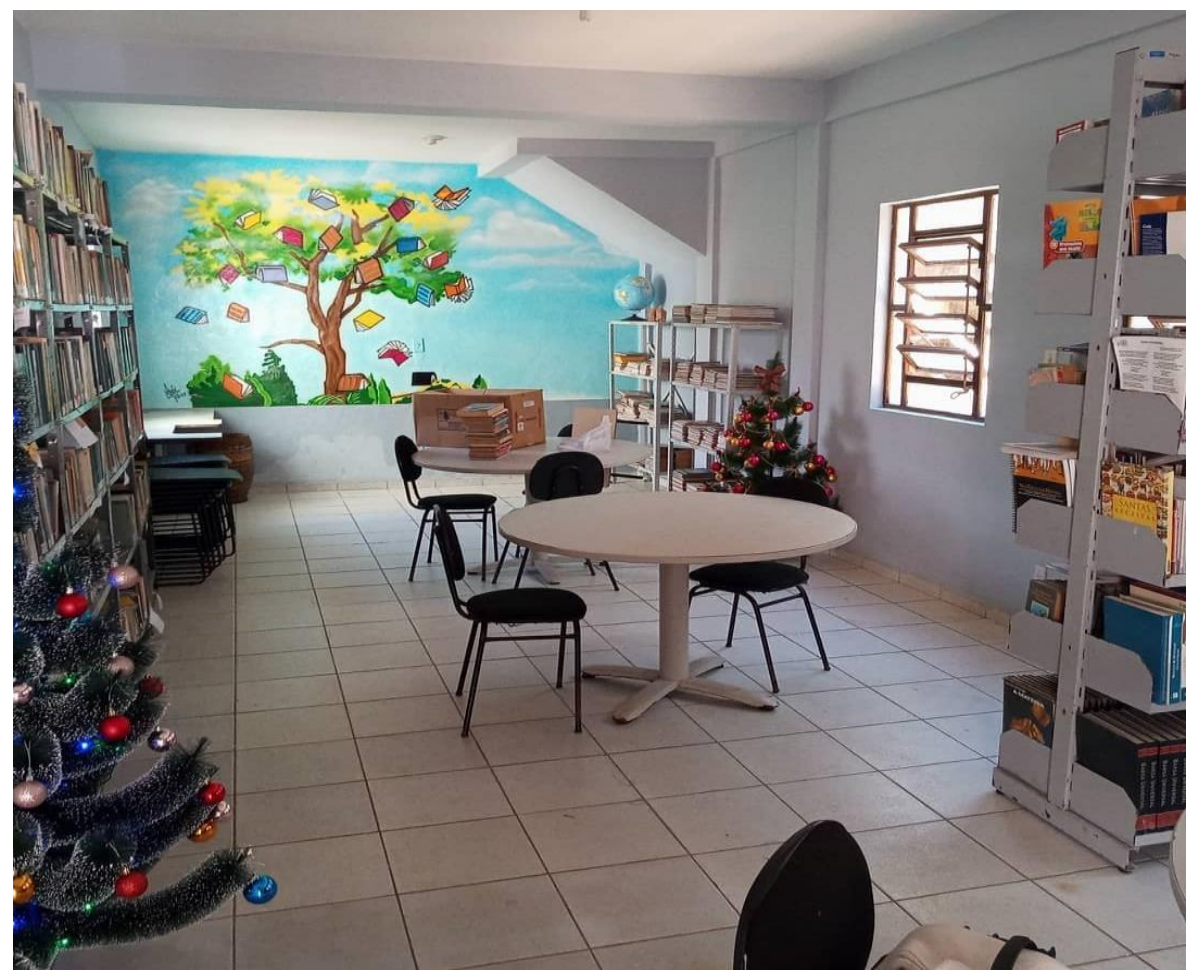

Fonte: elaborada pelo autor.

\section{CONSIDERAÇÕES FINAIS}

O Centro Cultural de Saramenha tem cumprido seu papel como espaço de convívio social, além do empréstimo de livros e incentivo à leitura, tem sido realizadas oficinas, auxílio no dever de casa e pesquisas e atividades recreativas. Também foram adquiridos novos livros e isso vem estimulando a leitura em todas as idades, com reflexos diretos nos resultados escolares, como confirmam os pais. Os bolsistas e voluntários apresentaram bom relacionamento e interação com a comunidade, assim como a mesma teve boa aceitação das atividades desenvolvidas no centro, isso pode ser percebido pelo grande número de frequentadores e a confiança que os pais e/ou responsáveis tem em deixar as crianças na biblioteca para realização das atividades sob supervisão dos bolsistas.

As atividades extensionistas são muito importantes, pois aproximam a comunidade da universidade e dos conhecimentos lá produzidos, em especial, nesse projeto, aos estudos e carreiras dentro das diversas engenharias ofertadas na UFOP. Além de ressaltar a importância do trabalho comunitário para a formação profissional e desenvolvimento da responsabilidade social dos estudantes.

\section{Agradecimentos}

$\mathrm{O}$ Centro Cultural agradece à Fundação Gorceix, grande parceira deste projeto e incentivadora das causas sociais, aos bolsistas e voluntários e à Pró-Reitoria de Extensão da UFOP pelo apoio e divulgação do trabalho. 


\title{
REFERÊNCIAS
}

FERNANDEZ, Cida; MACHADO, Elisa Campos; ROSA, Ester. O Brasil que lê: bibliotecas comunitárias e resistência cultural na formação de leitores. Centro de Cultura Luiz Freire. 2018. Disponível em:

http://www.serdigital.com.br/gerenciador/clientes/ceel/arquivos/167.pdf. Acesso em: 14 de abr. 2021.

FERNANDEZ, Maria Aparecida Arias; FINGER, Yasmin Wink. Bibliotecas comunitárias em rede: uma experiência de ressignificação de territórios. Disponível em:

http://repositorio.febab.org.br/files/original/24/3121/2156-2173-1-PB.pdf. Acesso em: 14 de abr. 2021.

LIMA, Marcele. Bibliotecas comunitárias: um mundo de possibilidades. Recife, 2020. Disponível em: https://www.anf.org.br/bibliotecas-comunitarias-um-mundo-de-

possibilidades/. Acesso em: 17 de abr. 2021.

MACHADO, Elisa Campos. Bibliotecas comunitárias como prática social no Brasil. 2008. 184f. Tese (Doutorado em Cultura e Informação) - Escola de Comunicações e Artes, Universidade de São Paulo, São Paulo, 2008. Disponível em:

http://www.teses.usp.br/teses-/disponiveis/27/27151/tde-07012009-172507/ptbr.php. Acesso em: 25 de abr. 2021.

\section{CULTURAL CENTER OF SARAMENHA: APPROACHING THE UNIVERSITY TO THE OURO PRETO COMMUNITY}

\begin{abstract}
The Cultural Center of Saramenha is the result of a joint action between the city hall municipality of Ouro Preto, UFOP - Department of Mining Engineering and the residents of the Saramenha neighborhood. The Center has a community library, registered at the National Library Foundation and has a collection of approximately 4000 books, updated at the disposal of the community, a hall for parties, a kitchen and a synthetic grass court. Space allows for community participation in diverse cultural activities, developed by volunteers and graduates from UFOP, which aim to combat informational exclusion and provides a place for coexistence, popular action, production and dissemination of culture and knowledge for the residents of the neighborhood and its surroundings. Such activities, such as encouraging reading, helping with homework and research, workshops dance classes, playful games, conversation circles, loans from books, games, tutoring and language classes attract the attention of the community and transform the Center into a pedagogical and educational support tool cultural diffusion for children, youth and adults. In this way the Cultural Center fulfills its role as a space for social interaction and amplifies the margins of learning of all involved. To residents, giving them opportunities previously not offered and undergraduates a rich opportunity for growth human and professional.
\end{abstract}

Keywords: extension, ingenuity, culture, community. 